\title{
Non-Singular Fields in General Relativity
}

\section{W. B. BONNOR}

\author{
Communicated by G. C. McVittie
}

\section{SUMMARY}

It is shown that the equations $R_{i k}=0$ in first approximation admit nonsingular solutions which are flat at spatial and temporal infinity.

An exact non-singular solution with cylindrical symmetry is obtained. A physical interpretation of this solution is given and it is shown to represent a gravitational wave which comes in from $r=\infty$ at $t=-\infty$, grows in intensity near $r=0$, reaching a maximum at about $t=0$, and then declines and finally disappears to $r=\infty$ at $t=\infty$. Space-time is non-singular everywhere, and flat at $r=\infty$ and at $t= \pm \infty$, but not at $z= \pm \infty$. It is possible that the infinite extension of the field in the $z$-direction may indicate the presence of material sources at $z= \pm \infty$.

1. Introduction. All published non-trivial solutions of the field equations of general relativity

$$
R_{i k}=0
$$

contain singularities. It was shown by EINstein \& Pauli [3] that there are no static non-singular solutions which represent mass, and the question has remained whether there exist any non-singular solutions at all.

The interest in non-singular solutions is due to the following considerations. In the fields known at present, the sources of gravitation are, so to speak, put into the theory from outside. They appear either as singularities, as in the Schwarzschild solution, or as an energy tensor, as in cosmology. In either case, the precise nature of the sources cannot be treated by general relativity alone. This is what one would expect, since all ordinary sources involve forces which are not gravitational, and so are outside the scope of general relativity.

It is therefore natural to ask whether the gravitational field, and therefore the nature of space-time, is always completely determined by the matter (or energy) present. If this is so, then, in the absence of matter, one would expect space-time to be completely flat. The only non-singular solution would be that referring to the empty world of special relativity. 
It would perhaps not be surprising if there were to exist solutions corresponding to isolated wave-packets of gravitational radiation. These would presumably be non-singular because the presence of gravitational radiation does not violate the field equations (1.1). One cannot be sure, however, that the theory will yield a model for an isolated packet of this kind; it may be that the solution will include also the sources of the packet, which will turn up as singularities. An isolated wave-packet, devoid of sources, is perhaps an artificial concept; but then, so is a single particle in an otherwise empty universe, and we know that the theory gives a solution in this case.

The energy of the gravitational field is not included in the energy tensor, and has, in fact, no covariant meaning. Hence if non-singular solutions of the field equations exist, we shall not be able to regard them as being "caused" by matter or energy. They will represent "pure" fields, that is to say, fields which contain their own sources, and in which the duality of field and particle does not arise. EINSTEIN spent many years trying to construct a more general field theory of this type, and it would be interesting if general relativity itself were found to admit such fields.

For these reasons it seems worth while to investigate further whether there are non-singular solutions of (1.1) and this paper is a contribution to this end. The main results of it are, first, in $\$ 2$, to show that there exist non-singular solutions of the equations in first approximation; and secondly, in §3, to produce an exact solution which is non-singular in a sense which is made precise in $\$ 4$. Later in the paper (\$5) the exact solution, which has a certain interest in its own right, is considered in more detail, and the work ends with a brief summary in $\$ 6$. To avoid raising false hopes, I should perhaps state at once that the exact solution which I give does not unambiguously answer the questions raised above, because one cannot be sure that the field which it represents is free from sources at infinity. However, the work described here seems, at least to me, to suggest that there may well be gravitational fields completely free of matter.

2. Non-singular solutions of the approximate equations. Let us consider the first approximation to the field equations (1.1) and see whether this admits non-singular solutions. Let us use an imaginary time coordinate $\left(x_{4}=i c t\right)$ and put

$$
g_{i k}=\delta_{i k}+\gamma_{i k}
$$

where the $\gamma_{i k}$ are small, so that products of the $\gamma_{i k}$ and of their derivatives may be neglected. The equations (1.1) then become

$$
\gamma_{i k, a a}+\gamma_{a a, i k}-\gamma_{i a, a k}-\gamma_{k a, a i}=0 .
$$

It is convenient to introduce the small quantities $\gamma_{i k}^{*}$ by

$$
\gamma_{i k}^{*}=\gamma_{i k}-\frac{1}{2} \delta_{i k} \gamma_{a a},
$$

and if we do this, the field equations (1.1) give

$$
\gamma_{i k, a a}^{*}-\gamma_{i a, a k}^{*}-\gamma_{k a, a i}^{*}-\frac{1}{2} \delta_{i k} \gamma_{a a, b b}^{*}=0
$$


If we make an infinitesimal transformation of coordinates,

$$
x_{i}^{\prime}=x_{i}+\xi_{i}\left(x_{i}\right),
$$

where the $\xi_{i}$ are small functions, we find that we can choose the coordinate system so that

$$
\gamma_{i a, a}^{*}=0 .
$$

The condition (2.4) does not completely determine the system of coordinates. If the $\gamma_{i k}$ satisfy (2.4), then $\gamma_{i k}^{\prime}$ obtained from them by a transformation of type (2.3), viz.

$$
\gamma_{i k}^{\prime}=\gamma_{i k}-\xi_{i, k}-\xi_{k, i},
$$

are also solutions of (2.4) provided that the $\xi_{i}$ satisfy the conditions

$$
\xi_{i, a a}=0 .
$$

If one can make the field of $\gamma_{i k}$ vanish by the addition of terms like those in (2.5) (that is to say, by an infinitesimal coordinate transformation) then the field is only an apparent one and has no physical significance.

From (2.2) and (2.4) it follows that the field equations in first approximation for empty space may be written

$$
\begin{aligned}
\gamma_{i k, a a}^{*} & =0, \\
\gamma_{i a, a}^{*} & =0 .
\end{aligned}
$$

This result is well-known. (See, for example Eddington [2] page 128.) Let us now see whether there are non-singular solutions of these equations.

Equation (2.6) is the ordinary wave equation (with imaginary time coordinate) for three-dimensional space, and it is known from the work of SyNGE [8] that it has non-singular solutions. Consider the equation in the form

$$
\frac{\partial^{2} \phi}{\partial x^{2}}+\frac{\partial^{2} \phi}{\partial y^{2}}+\frac{\partial^{2} \phi}{\partial z^{2}}-\frac{1}{c^{2}} \frac{\partial^{2} \phi}{\partial t^{2}}=0
$$

and the elementary solution

where

$$
\phi=\frac{1}{S},
$$

$$
S=\left(x-x_{0}\right)^{2}+\left(y-y_{0}\right)^{2}+\left(z-z_{0}\right)^{2}-c^{2}\left(t-t_{0}\right)^{2},
$$

$x_{0}, y_{0}, z_{0}, t_{0}$ being constants. Now let the constants be complex, and put

$$
S=A-i B,
$$

where $A$ and $B$ are real. Then

$$
\phi=\frac{A}{A^{2}+B^{2}}+i \frac{B}{A^{2}+B^{2}}=U+i V .
$$


Now $U$ and $V$ both satisfy (2.8) and it is easy to choose the constants so that $A$ and $B$ do not vanish together. By this means SyNGe obtains solutions which are regular throughout space-time, and which have also regular derivatives. As an example one may take

$$
\begin{aligned}
& U=\frac{R^{2}-c^{2} t^{2}+\beta^{2}}{\left(R^{2}-c^{2} t^{2}+\beta^{2}\right)^{2}+(2 \beta c t)^{2}}, \\
& V=\frac{-2 \beta c t}{\left(R^{2}-c^{2} t^{2}+\beta^{2}\right)^{2}+(2 \beta c t)^{2}},
\end{aligned}
$$

where $\beta$ is a real constant, and $R^{2}=x^{2}+y^{2}+z^{2}$.

We can now show that there exist regular solutions of (2.6) and (2.7). Put $x=x_{2}, y=x_{3}, z=x_{1}$, and take as the only non-zero $\gamma_{i k}^{*}$

$$
\gamma_{22}^{*}=U_{, 33}, \quad \gamma_{33}^{*}=U_{, 22}, \quad \gamma_{23}^{*}=-U_{, 32},
$$

where $U$ is given by (2.9). Then (2.6) and (2.7) are satisfied, and since $U$ and its derivatives are nowhere infinite (2.10) represents a solution of the approximate equations which is regular throughout space-time. It is easy to show also that this represents a real physical field since it cannot be reduced to zero by infinitesimal transformations in the way described previously. In terms of the $g_{i k}$, the solution (2.10) becomes

$$
\begin{aligned}
& g_{11}=1-\frac{1}{2}\left(U_{, 33}+U_{, 22}\right)=g_{44}, \\
& g_{22}=1+\frac{1}{2}\left(U_{, 33}-U_{, 22}\right), \\
& g_{33}=1-\frac{1}{2}\left(U_{, 33}-U_{, 22}\right), \\
& g_{23}=-U_{, 32} .
\end{aligned}
$$

One may check that all first and second derivatives of these $g_{i k}$ vanish at spatial and temporal infinity, so that the field is flat there.

Solutions of this type (which depend on three distinct $\gamma_{i k}^{*}$ ) are the simplest which can give non-singular fields which vanish at spatial infinity. Consider for example the simpler solutions which have only one non-zero $\gamma_{i k}^{*}$. Then from (2.7) these must either be static (e.g. $\gamma_{44}^{*} \neq 0$ ) or depend on less than three of the spatial coordinates $\left(e . g . \gamma_{11}^{*} \neq 0\right)$. In the former case the $\gamma_{i k}^{*}$ has to satisfy Laplace's equation and is singular if plausible conditions at spatial infinity are assumed, and in the latter the field does not tend to zero in all directions at spatial infinity. Similar considerations apply if only two of the $\gamma_{i k}^{*}$ are non-zero.

Instead of (2.10) one may take for the only non-zero $\gamma_{i k}^{*}$

$$
\gamma_{11}^{*}=U_{, 44}, \quad \gamma_{44}^{*}=U_{, 11}, \quad \gamma_{14}^{*}=-U_{, 41} .
$$

(In this case one must replace $c t$ by $-i x_{4}$ in (2.9).) This leads to the metric

$$
\begin{aligned}
& g_{11}=1+\frac{1}{2}\left(U_{, 44}-U_{, 11}\right), \\
& g_{22}=1-\frac{1}{2}\left(U_{.44}+U_{, 11}\right)=g_{33},
\end{aligned}
$$




$$
\begin{aligned}
& g_{44}=1-\frac{1}{2}\left(U_{, 44}-U_{, 11}\right), \\
& g_{14}=-U_{, 41} .
\end{aligned}
$$

Once again, this field is flat at spatial and temporal infinity. Since $x_{2}$ and $x_{3}$ occur only in the expression $x_{2}^{2}+x_{3}^{2}$ this suggests that there may be exact non-singular solutions with axial symmetry, and this expectation seems reasonable also on physical grounds.

More complicated non-singular solutions may be constructed in a similar way. One may easily verify that (2.6) and (2.7) are satisfied by each of the following arrays:

$$
\begin{aligned}
& \gamma_{11}^{*}=2 U_{, 2233} \quad \gamma_{12}^{*}=-U_{, 1233} \quad \gamma_{13}^{*}=-U_{, 1223} \\
& \gamma_{22}^{*}=2 U_{, 1133} \quad \gamma_{23}^{*}=-U_{, 1123} \\
& \gamma_{33}^{*}=2 U_{, 1122} \\
& \gamma_{14}^{*}=\gamma_{24}^{*}=\gamma_{34}^{*}=\gamma_{44}^{*}=0 ; \\
& \gamma_{11}^{*}=3 U_{, 223344} \quad \gamma_{12}^{*}=-U_{, 123344} \quad \gamma_{13}^{*}=-U_{, 122344} \quad \gamma_{14}^{*}=-U_{, 122334} \\
& \gamma_{22}^{*}=3 U_{, 113344} \quad \gamma_{23}^{*}=-U_{, 112344} \quad \gamma_{24}^{*}=-U_{, 112334} \\
& \gamma_{33}^{*}=3 U_{, 112244} \gamma_{34}^{*}=-U_{, 112234} \\
& \gamma_{44}^{*}=3 U_{, 112233}
\end{aligned}
$$

The task of finding an exact non-singular solution would be much simpler if any existed which had spherical symmetry. This is known not to be the case from Birkhoff's theorem, which states that any time-dependent solution of (1.1) with spherical symmetry may be transformed into Schwarzschild's solution. It is interesting to notice that the approximate equations do not suggest that there should be any regular solutions with spherical symmetry. To prove this we start from the fact that any spherically symmetric metric may be put in the form

$$
d s^{2}=A\left(d x^{2}+d y^{2}+d z^{2}\right)+B d x_{4}^{2},
$$

where $A$ and $B$ are functions of $R$ and $x_{4}$ (Kustannhermo [5]). This is true whether or not the field is weak. The weak-field equations in the form (2.1) then give a set of equations of which the following are typical

$$
\begin{array}{ll}
2 G_{11}=A_{, a a}+(A+B)_{, 11} & =0, \\
2 G_{12}=(A+B)_{, 12} & =0, \\
2 G_{14}=2 A_{, 14} & =0, \\
2 G_{44}=B_{, a a}+3 A_{, 44}-B_{, 44} & =0 .
\end{array}
$$

One finds without difficulty that

$$
A=1+\frac{2 m}{R}+\alpha x_{4}, \quad B=1-\frac{2 m}{R}+\beta x_{4},
$$


where $m, \alpha$, and $\beta$ are constants. This solution is obviously singular. The fact that the approximate solutions do not mislead us in the spherically symmetric case allows one to hope that they may not do so in the other cases, and that the existence of non-singular solutions of the approximate equations may be an indication that regular exact solutions also exist.

To find exact non-singular solutions which are flat at infinity is evidently very difficult. Even supposing that non-singular fields with axial symmetry exist, the $g_{i k}$ must be functions of three variables, and the field equations become very complicated. In the next section I undertake the simpler task of looking for non-singular solutions in the case of cylindrical symmetry, in which the $g_{i k}$ depend only on $r$ and $t$, where $r$ is a radial coordinate measured from the axis of symmetry. Of course, for such solutions one knows in advance that the field cannot tend to zero as the axial coordinate $(z)$ tends to infinity, since the $g_{i k}$ are independent of $z$. We shall have to bear in mind this fact when we discuss the physical significance of the solution, and to consider whether it constitutes a "singularity" of the field.

3. An exact non-singular solution with cylindrical symmetry. Let $z$ be the coordinate measured along the axis of symmetry, $r$ and $\theta$ the radial and angular coordinates, and $t$ the time. Then, for a field depending only on $r$ and $t$, it was shown by EINSTEIn \& Rosen [4] that the metric is

$$
d s^{2}=-e^{\rho} d z^{2}-e^{\lambda} d r^{2}-r^{2} e^{-\rho} d \theta^{2}+e^{\lambda} d t^{2},
$$

where $\rho \equiv \rho(r, t), \lambda \equiv \lambda(r, t)$, and that the field equations reduce to

$$
\begin{aligned}
\rho_{r r}+r^{-1} \rho_{r}-\rho_{t t} & =0, \\
\lambda_{r}+\rho_{r} & =\frac{1}{2} r\left(\rho_{r}^{2}+\rho_{t}^{2}\right), \\
\lambda_{t}+\rho_{t} & =r \rho_{r} \rho_{t} .
\end{aligned}
$$

Here a suffix $r$ or $t$ means differentiation with respect to $r$ or $t$.

Equation (3.2) is the equation for cylindrical waves in Euclidean space. Einstein \& Rosen, and later Rosen [7], obtained solutions of it which lead to particular cases of the metric (3.1) corresponding to progressive or stationary gravitational waves; these solutions contain a singularity along the axis of $z$, presumably representing the source of the waves.

I shall now obtain a non-singular solution of (3.2) by adapting the procedure used by Synge in the case of the three-dimensional wave equation (2.10). Consider the following function

$$
\frac{4 b}{\left[r^{2}-(t-i a)^{2}\right]^{\frac{1}{2}}}
$$

where $a, b$ are real constants. This satisfies (3.2), and on separating it into real and imaginary parts (both of which satisfy (3.2)), we obtain

$$
2 \sqrt{2} b\left[\frac{u+\sqrt{u^{2}+v^{2}}}{u^{2}+v^{2}}\right]^{\frac{1}{2}}-2 i \sqrt{2} b\left[\frac{\sqrt{u^{2}+v^{2}}-u}{u^{2}+v^{2}}\right]^{\frac{1}{2}},
$$


where

$$
u=r^{2}-t^{2}+a^{2}, \quad v=2 a t .
$$

In (3.5) positive square roots are to be taken throughout.

Let us now take for the function $\rho$ in the metric (3.1) the real part of (3.5):

$$
\rho=2 \sqrt{2} b\left[\frac{u+\sqrt{u^{2}+v^{2}}}{u^{2}+v^{2}}\right]^{\frac{1}{2}} .
$$

From the values of $u$ and $v$ given in (3.6) it is clear that $\rho$ in (3.7) is bounded for all $r$ and $t$.

To find $\lambda$ we have to integrate the equations (3.3) and (3.4), which are known to be compatible as they come from the field equations (1.1). The result is

$$
\begin{aligned}
\lambda=-2 \sqrt{2} b\left[\frac{u+\sqrt{u^{2}+v^{2}}}{u^{2}+v^{2}}\right]^{\frac{1}{2}}-\frac{2 b^{2} r^{2}\left(u^{2}-v^{2}\right)}{\left(u^{2}+v^{2}\right)^{2}} & \\
& +\frac{b^{2}}{a^{2}}\left[\frac{r^{2}-a^{2}-t^{2}}{\left(u^{2}+v^{2}\right)^{\frac{3}{3}}}+1\right] .
\end{aligned}
$$

For the arbitrary constant which arises in the integration we have chosen the value $b^{2} / a^{2}$ for reasons which will become clear later.

The functions $\rho$ and $\lambda$ given by (3.7) and (3.8) give a non-singular metric (3.1), as will be explained in $\$ 4$.

4. The regularity of the solutions. There is in general relativity no accepted definition of a physical singularity. One plausible definition of a non-singular point is that it is one at which natural coordinates can be introduced by a coordinate transformation. A natural coordinate system at a given point $P$ is one in which the $g_{i k}$ take Galilean values at $P$, and in which the first derivatives of the $g_{i k}$ vanish at $P$ (EDdington, [2], page 76). Physically, this definition means that at a non-singular point it is possible for an observer to choose his coordinates so that test particles in his immediate neighbourhood move in straight lines; thus the effects of gravitation at the point may be removed by a coordinate transformation.

Given a metric $g_{i k}$, sufficient conditions for the introduction of natural coordinates at $P$ are

(i) $g$, the determinant of the $g_{i k}$, is non-zero;

(ii) $g_{i k}$ and their first derivatives are finite and continuous at $P$;

(iii) the second derivatives of $g_{i k}$ are finite and continuous at $P$.

These conditions are not necessary; in fact the third condition could be dispensed with completely as far as the introduction of natural coordinates is concerned; I keep it here because the three conditions together ensure that the RiemannChristoffel tensor is finite and continuous, which is an additional safeguard against singularity. Even the first two conditions are not necessary, as one can readily appreciate if one recalls that the metric for flat space-time in polar coordinates does not satisfy them at all points. 
To verify the regularity of the solution in $\$ 3$ let us transform to pseudoCartesian coordinates by putting

$$
x=r \cos \theta, \quad y=r \sin \theta, \quad z=z, \quad t=t .
$$

The result is

$$
\begin{aligned}
d s^{2}=-e^{p} d z^{2}-r^{-2}\left[d x^{2}\left(x^{2} e^{\lambda}+y^{2} e^{-\rho}\right)\right. & +d y^{2}\left(y^{2} e^{\lambda}+x^{2} e^{-\rho}\right) \\
& \left.+2 x y d x d y\left(e^{\lambda}-e^{-\rho}\right)\right]+e^{\lambda} d t^{2},
\end{aligned}
$$

where $\rho$ and $\lambda$ are given by (3.7) and (3.8). Then one can check by straightforward calculation that the conditions (4.1) are satisfied for all points of spacetime, that is to say, throughout the region bounded by

$$
x= \pm \infty, \quad y= \pm \infty, \quad z= \pm \infty, \quad t= \pm \infty \text {. }
$$

Therefore the metric (4.2) is a solution of the field equations which is everywhere regular in the foregoing sense.

One also finds that the components of the Riemann-Christoffel tensor for the metric (4.2) tend to zero as $x, y$, and $t$ tend to infinity, together or separately. As $z$ tends to infinity, these components do not all tend to zero. This is, of course, due to the nature of the symmetry which was assumed at the start. The solution is the same on any hyperplane $z=$ constant. I shall return to this point in the next section.

It will be convenient to distinguish between those non-singular fields which tend to flatness as the four coordinates tend to infinity and those which do not. By a non-singular (or regular) field I shall mean one in which every point is nonsingular (including points at spatial and temporal infinity). By a localized nonsingular field will be meant a non-singular one which has the additional property that the metric tends to flatness $\left(B_{i k l m}\right.$ tends to zero) as any one or more of the four coordinates tends to infinity.

With this terminology we may say that the solutions of $\$ 2$ are localized nonsingular solutions of the approximate equations; and that the exact solution of $\S 3$ is non-singular but not localized.

5. The physical significance of the exact solution. It is clear that the solution given in $\$ 3$ does not correspond to a field of ordinary matter. The static material field with the same spatial symmetry is that of a line-mass placed along the axis of $z$, and the metric corresponding to it is (Levi-Crvita, [6]):

$$
d s^{2}=-\left(\frac{r}{r_{0}}\right)^{2 m^{2}-2 m}\left(d z^{2}+d r^{2}\right)-r^{2}\left(\frac{r_{0}}{r}\right)^{2 m} d \theta^{2}+\left(\frac{r}{r_{0}}\right)^{2 m} d t^{2},
$$

where $r_{0}$ and $m$ are constants. To compare our solution with this, we may put $t=$ constant in the $g_{i k}$; for example, if we put $t=0$ in (3.6), (3.7), and (3.8), we find

$$
\rho=\frac{4 b}{\sqrt{r^{2}+a^{2}}}
$$




$$
\lambda=-\frac{4 b}{\sqrt{r^{2}+a^{2}}}+\frac{2 b^{2} r^{4}}{a^{2}\left(r^{2}+a^{2}\right)^{2}}
$$

Then, for large $r$, we have

$$
\begin{aligned}
& g_{11}=-\left[1+\frac{4 b}{r}+O\left(\frac{1}{r^{2}}\right)\right], \\
& g_{44}=\text { constant } \times\left[1-\frac{4 b}{r}+O\left(\frac{1}{r^{2}}\right)\right] .
\end{aligned}
$$

Evidently these values of $g_{i k}$ do not represent the field of a line-mass.

Since the function $\rho$ in the metric (3.1) satisfies the wave equation (3.2), one is naturally tempted to refer to the solution of $\$ 3$ as a gravitational wave. I shall allow myself to yield to this temptation in spite of the fact that the equation (3.2) depends on the coordinate system so that this classification is not a covariant one.

As $r$ tends to infinity, the components of the Riemann-Christoffel tensor (for the metric in the form (4.2)) tend to zero like $r^{-3}$, provided $t$ is finite. Similarly if $r$ is finite and $t$ tends to $\pm \infty$, these components tend to zero like $t^{-3}$. On the surface $r= \pm t$, however, the components tend to zero like $r^{-\frac{1}{2}}$ (or $t^{-\frac{1}{2}}$ ). (For the convenience of anyone who may wish to study solutions of the metric (3.1) $I$ give in an Appendix the non-zero components of the Riemann-Christoffel tensor in terms of $\rho$ and $\lambda$.)

Let us study the history of the field on the axis of symmetry $r=0$. We find there

$$
\rho=-\lambda=\frac{4 b a}{a^{2}+t^{2}}
$$

Thus the difference of the $g_{i k}$ from Galilean values is greatest when $t=0$. If we make $a$ large, we prolong the time during which $|\rho|$ and $|\lambda|$ are approximately equal to their maximum values:

$$
|\rho| \sim 4\left|\frac{b}{a}\right|
$$

Thus we may speak of $a$ as giving a measure of the "lifetime" of the wave, in the sense that for a period of about $a$, the field is near its maximum intensity as measured, for example, by the invariant $B$ referred to below. The constant $b$, on the other hand, is related to the intensity of the field since it determines the field for large $r$, from (5.1).

Instead of considering the values of the $g_{i k}$, it would be better to study the history of an invariant of the field. The simplest one available is

$$
B=B_{k l m}^{i} B_{i}^{k l m},
$$

which is extremely complicated for this solution. If one calculates $B$ on $r=0$ one finds that the conclusions of the previous paragraph are confirmed in a 
general way, although the situation is rather more complex than that suggested by the simple function (5.2). Briefly, the graph of $B$ as a function of $t$ (on $r=0$ ) shows a finite number of maxima and minima, one of which occurs at $t=0$. Naturally, $B$ tends to zero (like $t^{-8}$ ) as $t$ tends to $\pm \infty$.

We are now in a position to give a rough picture of the history of the solution. It represents a cylindrical wave which comes in from $r=\infty$ at $t=-\infty$, grows in intensity near $r=0$, reaching its greatest intensity there at about time $t=0$. It then declines and finally disappears to $r=\infty$ at $t=+\infty$. The values of $g_{11}$ and $g_{44}$ for large $r$ are given, for all finite $t$, by (5.1).

The solution represents a non-localized regular field, in the sense of $\$ 4$, and we have still to decide on the physical significance of the fact that it extends to infinity in the $z$-direction. In particular we should like to know whether this represents material sources of the wave at $z= \pm \infty$. If this is not the case, then we have a solution without material sources, since there are none in the finite region of the field, or at $r=\infty$ or $t= \pm \infty$.

Unfortunately I have not been able to find any clear answer to this question. In another known solution of general relativity in which there are sources at infinity - the uniform gravitational field-there are singularities in the metric which correspond in an identifiable way to the mass particles which give rise to the field (BonNor [1]). This might suggest that a source at infinity, like a source anywhere else, appears as a singularity. On the other hand, it is possible that the most natural explanation of the field described here may be in terms of oscillations of material particles at $z= \pm \infty$. The argument which suggests this is as follows.

If we regard the constant $b$ in the solution as small we may take as the first approximation to $(4.2)$

$$
\begin{aligned}
& g_{11}=-1-\rho, \\
& g_{22}=g_{33}=-g_{44}=-1+\rho, \\
& g_{r s}=0 \quad(r \neq s),
\end{aligned}
$$

since these differ from the exact $g_{i k}$ by terms of order $b^{2}$. Returning now to the approximate equations of $\S 2$, we see that the field (5.3) can be represented by the single function $\gamma_{11}^{*}$, which satisfies

$$
\gamma_{11,1}^{*}=0
$$

in accordance with the symmetry of the solution.

Let us suppose for the moment that in the approximate equations we have an energy tensor $T_{i k}$, so that equations (2.6) and (2.7) become

$$
\begin{aligned}
& \gamma_{i k, a a}^{*}=-16 \pi T_{i k}, \\
& \gamma_{i a, a}^{*}=0 .
\end{aligned}
$$


The solution of (5.4) is given by the retarded potential:

$$
\gamma_{i k}^{*}=4 \int \frac{\left[T_{i k}\right]}{R} d v
$$

Suppose that the sources of the field consist of matter of density $\rho$ oscillating with velocity $w$ parallel to the $z$-axis. Then we have $T_{11}=\rho w^{2}$, and we obtain $\gamma_{11}^{*}$ in terms of this from (5.6). This function $\gamma_{11}^{*}$ will depend on $z$, and there will be other non-zero components of $\gamma_{i k}^{*}$ in accordance with (5.5). However, it might be possible, by taking an appropriate function for $w(t)$, by allowing it to be large, and by making the oscillating particles tend to $z= \pm \infty$, to generate a field which becomes, in the limit, similar to (5.3).

This argument is, of course, extremely tentative; but it does perhaps suggest that it would be unsafe without further investigation to regard the exact solution of $\$ 3$ as representing a field without material sources.

6. Conclusion. Although it is not possible to draw from this work any definite conclusion about the existence of localized non-singular solutions of equations (1.1), the fact that such solutions exist in the first approximation is interesting. Further, it may be possible to find the nature of the second approximation for solutions of this type, in the following way.

Let us consider a solution of the field equations (1.1) in a series of terms of decreasing order of magnitude:

$$
g_{i k}=\delta_{i k}+{ }_{1} h_{i k}+{ }_{2} h_{i k}+\cdots .
$$

Take

$$
{ }_{1} h_{i k}=\lambda_{1} \gamma_{i k}
$$

where ${ }_{1} \gamma_{i k}$ is one of the localized non-singular solutions given in $\S 2$, and $\lambda$ is a constant. We know that ${ }_{1} \gamma_{i k}$ is bounded throughout space-time and that its derivatives are bounded. Then, using the field equations, we can solve for ${ }_{2} h_{i k}$ in terms of the retarded potentials corresponding to a source function which depends on the products of the ${ }_{1} \gamma_{i k}$ and their derivatives. This will give

$$
{ }_{2} h_{i k}=\lambda^{2}{ }_{2} \gamma_{i k} \text {, }
$$

where the ${ }_{2} \gamma_{i k}$ are, like the ${ }_{1} \gamma_{i k}$, independent of $\lambda$. If we can prove that ${ }_{2} \gamma_{i k}$ and their derivatives are bounded, and that they vanish appropriately at infinity, this will give a solution which is non-singular and localized as far as the second approximation. By continuing this process it might even be possible to prove the existence of rigorous localized non-singular solutions, since if all the ${ }_{n} \gamma_{i k}$ are bounded, $\lambda$ can be chosen to make the series converge at all points in space-time.

The exact solution of $\$ 3$ is certainly non-singular. It is not localized, but this, of course, is a necessary result of the cylindrical symmetry assumed at the start. 
The fact that one obtains a non-singular solution in this case suggests that one might find exact localized non-singular solutions if one relaxed the symmetry conditions, in agreement with the results of the theory in the first approximation.

\section{Appendix}

The Riemann-Christoffel tensor for the metric (3.1). If one takes the Riemann-Christoffel tensor in the form

$$
B_{i j k l}=-\Gamma_{j l}^{a}[i k, a]+\Gamma_{j k}^{a}[i l, a]+\frac{1}{2}\left(g_{i l, j k}+g_{j k, i l}-g_{j l, i k}-g_{i k, i l}\right),
$$

it has twenty independent components. In the case of the metric (3.1) the following eight of these do not vanish:

$$
\begin{aligned}
& B_{1212}=-r^{-2} e^{2 \rho} B_{3434}=\frac{1}{4} e^{\rho}\left(2 \rho_{r r}+\rho_{r}^{2}-\lambda_{r} \rho_{r}-\lambda_{t} \rho_{t}\right), \\
& B_{1313}=-r^{2} e^{-2 \lambda} B_{2424}=\frac{1}{4} r^{2} e^{-\lambda}\left(2 r^{-1} \rho_{r}-\rho_{r}^{2}+\rho_{t}^{2}\right), \\
& B_{1414}=-r^{-2} e^{2 \rho} B_{2323}=\frac{1}{4} e^{\rho}\left(2 \rho_{t t}+\rho_{t}^{2}-\rho_{t} \lambda_{t}-\rho_{r} \lambda_{r}\right), \\
& B_{1214}=r^{-2} e^{2 \rho} B_{2334}=\frac{1}{4} e^{\rho}\left(2 \rho_{r t}+\rho_{r} \rho_{t}-\lambda_{r} \rho_{t}-\lambda_{t} \rho_{r}\right) .
\end{aligned}
$$

\section{References}

[1] Bonnor, W. B., Proceedings of the Physical Society (A) 66 (1953) p. 145.

[2] Eddington, A. S., "The Mathematical Theory of Relativity", Cambridge University Press, (1952).

[3] Einstern, A. \& Paulr, Annals of Mathematics 44 (1943) p. 131.

[4] Einstein, A. \& Rosen, Journal of the Franklin Institute 223 (1937) p. 43.

[5] Kustaanheimo, P., Proceedings of the Edinburgh Mathematical Society (2) 9 (1953) p. 13.

[6] Levi-Civita, T., Atti Accademia dei Lincei. Rendiconti 28 (1919) I p. 101.

[7] Rosen, N., Bulletin of the Research Council of Israel 3 (1954) p. 328.

[8] Synge, J. L., "Relativity: The Special Theory", North-Holland Publishing Company, Amsterdam, (1956). 